\title{
Association of traumatic brain injury in childhood and attention-deficit/hyperactivity disorder: a population-based study
}

\author{
Ling-Yu Yang ${ }^{1}$, Chao-Ching Huang ${ }^{2,3}$, Wen-Ta Chiu ${ }^{4}$, Li-Tung Huang ${ }^{5}$, Wei-Cheng Lo ${ }^{6}$ and Jia-Yi Wang ${ }^{1,7}$
}

\begin{abstract}
BACKGROUND: We evaluated the risk of attention-deficit hyperactivity disorder (ADHD) following childhood traumatic brain injury (TBI).

METHODS: Using Taiwan's National Health Insurance Research Database, we included 10,416 newly diagnosed TBI children (aged $\leq 12$ y) between 2001 and 2002 and 41,664 children without TBI, who were frequency matched by sex, age, and year of the index medical service with each TBI child, as controls. Children who had been diagnosed with ADHD prior to their medical service index were excluded. Each individual was followed for 9 y to identify ADHD diagnosis. We also compared the ADHD risk in children who were treated for fractures but not TBI as sensitivity analysis.
\end{abstract}

RESULTS: During the 9-y follow-up period, children with TBI had a higher ADHD risk (adjusted hazard ratio $(A H R)=1.32,95 \%$ confidence interval $(\mathrm{Cl})=1.19,1.45$ ) than did those without TBI. Furthermore, children with mild and severe TBI had higher AHRs for ADHD than did those without TBI (AHR $=1.30 ; 95 \%$ $\mathrm{Cl}=1.10,1.53$; and $\mathrm{AHR}=1.37 ; 95 \% \mathrm{Cl}=1.22,1.55)$. However, no significant association was observed between fractures and ADHD.

CONCLUSION: TBI in childhood is associated with a greater likelihood of developing ADHD.

$\mathbf{T}$ raumatic brain injury (TBI) is the leading cause of death and disability in children and often results in persistent behavioral disturbances and neurocognitive deficits in attention, learning, and memory (1-3). Children with cognitive impairment following TBI impose a great economic and social burden on their families and communities. In addition, these children are more likely to have slower psychosocial development and exhibit poor academic achievement. Therefore, the relationship between TBI and neuropsychiatric consequences has become a crucial research topic.

Attention-deficit-hyperactivity disorder (ADHD), a neurodevelopmental disorder, is associated with impulsivity, excessive talking, difficulty in sustaining attention, being easily distracted, and injuries (4-6). Studies have reported a relationship between ADHD and childhood TBI (7-10). Unlike other diseases resulting from various pathogenic mechanisms, TBI is caused by a complex interplay among damages occurring in the neuroanatomy, neurochemistry, and neurophysiology because of primary and secondary processes (11). A meta-analytic review showed that neurocognitive outcomes after pediatric TBI have a dose-response relationship with injury severity. Babikian and colleagues found that patients with severe TBI exhibited poorer performance in intelligence quotient tests, executive functioning, processing speed, attention maintenance, verbal immediate memory, and delayed memory, whereas patients with mild TBI exhibited no change (12). In addition, several studies have suggested that children with moderate to severe TBI are significantly more likely to develop ADHD symptoms than are those with mild TBI $(8,13-17)$. A longitudinal birth cohort study reported no association between preschool mild TBI and ADHD after adjusting for potential covariates (18). Nevertheless, a prospective cohort study reported that children with mild TBI have a higher risk of hyperactivity in the first year after injury (19). Nevertheless, a prospective cohort study reported children with mild TBI were associated higher risk for hyperactivity in the first year after injury (20). However, the restricted sample size and relatively brief follow-up period may cause difficulty in generalizing results and reflecting the true incidence. Therefore, the relationship between TBI and ADHD remains controversial in children. Using a nationwide population-based longitudinal study and including an injury severity range from mild to severe would facilitate clarifying the relationship between TBI and $\mathrm{ADHD}$ and developing a more satisfactory care procedure for children with TBI.

Earlier studies have shown that children diagnosed or prediagnosed with ADHD are more likely to have suffered from injuries including TBI, fractures, or burns $(21,22)$. In this study, we evaluated the relationship between TBI and ADHD

\footnotetext{
'Graduate Institute of Medical Sciences, College of Medicine, Taipei Medical University, Taipei, Taiwan; ${ }^{2}$ Department of Pediatrics, National Cheng Kung University Hospital and College of Medicine, Tainan, Taiwan; ${ }^{3}$ Department of Pediatrics, College of Medicine, Taipei Medical University, Taipei, Taiwan; ${ }^{4}$ Graduate Institute of Injury Prevention and Control, Taipei Medical University, Taipei, Taiwan; ${ }^{5}$ Department of Pediatrics, Kaohsiung Chang Gung Memorial Hospital and Chang Gung University, College of Medicine, Kaohsiung, Taiwan; ${ }^{6}$ Graduate Institute of Epidemiology and Preventive Medicine, College of Public Health, National Taiwan University, Taipei, Taiwan; ${ }^{7}$ Department of Physiology, School of Medicine, College of Medicine, Taipei Medical University, Taipei, Taiwan. Correspondence: Jia-Yi Wang (jywang2010@tmu.edu.tw) 
through sensitivity analysis to compare the ADHD risk in children receiving index treatment for fractures but not TBI. We conducted a retrospective, population-based cohort study by using a nationwide population-based data set to examine the long-term ADHD risk in children after TBI or fractures. Furthermore, we predicted the cause-specific risk of post-TBI ADHD among different TBI patterns on the basis of clinical characteristics. The findings may facilitate developing an integrated healthcare strategy for children with TBI.

\section{RESULTS}

The distribution of sociodemographic characteristics for children with and without TBI is shown in Table 1. Of 52,080 children aged $\leq 12$ y enrolled between 2001 and 2002, 10,416 children newly diagnosed with TBI were included as cases and 41,664 children without TBI as controls. The mean age of the 52,080 children was $5 \mathrm{y}$ and $7 \mathrm{mo}$ (SD $=3 \mathrm{y}$ and $4 \mathrm{mo}$ ), and $60.9 \%$ of them were male. After the cases and controls were matched by age and sex, the children with TBI were significantly more likely to have self-employed (farmer, craftsman, and fisher) parents $(P<0.001)$ and reside in less urbanized areas $(P<$ $0.001)$ compared with the children without TBI. For sensitivity analysis, we included 2,179 children who received treatment for fractures but not TBI between 2001 and 2002 in the fracture group as cases and 8,716 children without fractures or TBI in the nonfracture group as controls (Supplementary Table S1

Table 1. The sociodemographic characteristics of children with traumatic brain injury (TBI) and non-TBI controls in Taiwan, 2001$2002(n=52,080)$

\begin{tabular}{|c|c|c|c|c|c|}
\hline \multirow[b]{2}{*}{ Variables } & \multicolumn{2}{|c|}{$\begin{array}{c}\text { Children with TBI } \\
\quad N=10,416\end{array}$} & \multicolumn{2}{|c|}{$\begin{array}{c}\text { Non-TBI controls } \\
N=41,664\end{array}$} & \multirow[b]{2}{*}{$P$ value } \\
\hline & No. & $\%$ & No. & $\%$ & \\
\hline Age & & & & & - \\
\hline$\leq 4 y$ & 4,594 & 44.1 & 18,376 & 44.1 & - \\
\hline $5-8 y$ & 3,277 & 31.5 & 13,108 & 31.5 & - \\
\hline $9-12 y$ & 2,545 & 24.4 & 10,180 & 24.4 & - \\
\hline Sex & & & & & - \\
\hline Male & 6,340 & 60.9 & 25,360 & 60.9 & - \\
\hline Female & 4,076 & 39.1 & 16,304 & 39.1 & - \\
\hline Urbanization level & & & & & $<0.001$ \\
\hline 1 (highest) & 2,636 & 25.3 & 11,936 & 28.7 & \\
\hline 2 & 2,801 & 26.9 & 12,000 & 28.8 & \\
\hline 3 & 2,114 & 20.3 & 7,926 & 19.0 & \\
\hline 4 & 1,594 & 15.3 & 5,514 & 13.2 & \\
\hline 5 (lowest) & 1,271 & 12.2 & 4,288 & 10.3 & \\
\hline Parental occupation & & & & & $<0.001$ \\
\hline Manager, white collar & 656 & 6.3 & 3,734 & 9.0 & \\
\hline $\begin{array}{l}\text { Employee or manual } \\
\text { worker }\end{array}$ & 6,835 & 65.6 & 28,512 & 68.4 & \\
\hline $\begin{array}{l}\text { Self-employed (farmer, } \\
\text { craftsman, and fisher) }\end{array}$ & 1,699 & 16.3 & 4,948 & 11.9 & \\
\hline Others & 1,226 & 11.8 & 4,470 & 10.7 & \\
\hline
\end{tabular}

online). No differences were observed in urbanization level and parental occupation between the fracture and nonfracture groups.

Table 2 shows crude and covariate-adjusted hazard ratios (HRs) and their 95\% confidence intervals (CIs) for children who developed ADHD during the 9-y follow-up period. Compared with the children without TBI, those with TBI had a significantly higher incidence of ADHD (5.42 vs. $4.01 \%$ ) during the 9-y period following their medical service index use. HRs were calculated after excluding children who had previous history of preterm births, congenital anomaly, mental illness, epilepsy or cerebral palsy. Conditional Cox proportional hazard regression analysis revealed that TBI was significantly associated with ADHD (adjusted HR (AHR) $=1.24,95 \%$ CI $=1.14,1.37)$. After adjustment for children's age, parental occupation, and urbanization level, the results showed that TBI was significantly associated with ADHD (AHR $=1.32,95 \% \mathrm{CI}$ $=1.19,1.45)$. Furthermore, sensitivity analysis revealed that the ADHD incidence did not differ between the children with fractures and those without fractures (2.89 vs. $2.96 \%)$, and no significant association was observed between fracture and ADHD $(\mathrm{AHR}=1.03,95 \% \mathrm{CI}=0.77,1.37)$ (Supplementary Table S2 online).

The median time for children diagnosed with ADHD during the follow-up period to develop ADHD after their medical service index use was $4 \mathrm{y}$ and $8 \mathrm{mo}$ ( $4 \mathrm{y}$ and $7 \mathrm{mo} ; 4 \mathrm{y}$ and $9 \mathrm{mo}$ for children with TBI and those without TBI, respectively). The log-rank test showed that the children with TBI had a significantly higher 9 -y cumulative ADHD incidence $(P<0.001)$ than did those without TBI. The 9-y cumulative incidence curve is shown in Figure 1.

The AHRs for ADHD for subgroups stratified by age, sex, types of brain injury, and subtypes of severe brain injury are presented in Table 3. After adjustment for age, parental occupation, and urbanization level, conditional Cox proportional hazard regression analysis showed that children with TBI onset age, at aged $\leq 4$ and 5-8 y were significantly associated with a higher risk of ADHD compared with those without TBI $(\mathrm{AHR}=1.39,95 \% \mathrm{CI}=1.23,1.58$; $\mathrm{AHR}=1.23,95 \% \mathrm{CI}$ $=1.03,1.48$, respectively). Boys and girls with TBI showed a significantly increased ADHD risk compared with that in the children without TBI $(\mathrm{AHR}=1.32,95 \% \mathrm{CI}=1.18,1.47$; $\mathrm{AHR}=1.31,95 \% \mathrm{CI}=1.03,1.65$, respectively). Children with

Table 2. Crude and covariate-adjusted HRs for ADHD among the sampled patients during the 9 -y follow-up period $(n=52,080)$

\begin{tabular}{|c|c|c|c|c|c|c|}
\hline \multirow[b]{2}{*}{ ADHD occurrence } & \multicolumn{2}{|c|}{$\begin{array}{c}\text { Total } \\
\text { sample }\end{array}$} & \multicolumn{2}{|c|}{$\begin{array}{c}\text { Children with } \\
\text { TBI }\end{array}$} & \multicolumn{2}{|c|}{$\begin{array}{l}\text { Non-TBI } \\
\text { controls }\end{array}$} \\
\hline & $N$ & $\%$ & $N$ & $\%$ & $N$ & $\%$ \\
\hline Yes & 2,237 & 4.30 & 565 & 5.42 & 1,672 & 4.01 \\
\hline No & 49,843 & 95.70 & 9,851 & 94.58 & 39,992 & 95.99 \\
\hline Crude HR (95\% Cl) & \multicolumn{2}{|c|}{ - } & \multicolumn{2}{|c|}{$1.24^{*}(1.14,1.37)$} & \multicolumn{2}{|c|}{1.00 (reference) } \\
\hline Adjusted HR $(95 \% \mathrm{Cl})^{\mathrm{a}}$ & \multicolumn{2}{|c|}{-} & \multicolumn{2}{|c|}{$1.32^{*}(1.19,1.45)$} & \multicolumn{2}{|c|}{1.00 (reference) } \\
\hline
\end{tabular}




\section{Articles | Yangetal.}

mild and severe TBI had 1.30-fold (95\% CI $=1.10,1.53)$ and 1.37 -fold $(95 \% \mathrm{CI}=1.22,1.55)$ increased ADHD risks during the 9 -y follow-up period compared with that of the children without TBI, respectively. However, children with skull bone fractures showed no significant increase in the ADHD risk compared with that in the children without TBI. Furthermore, according to types of severe brain injury, the ADHD risk was significantly increased in children with brain contusion or subdural haemorrhage $(\mathrm{SDH})(\mathrm{AHR}=1.61,95 \% \mathrm{CI}=1.22,2.13$;

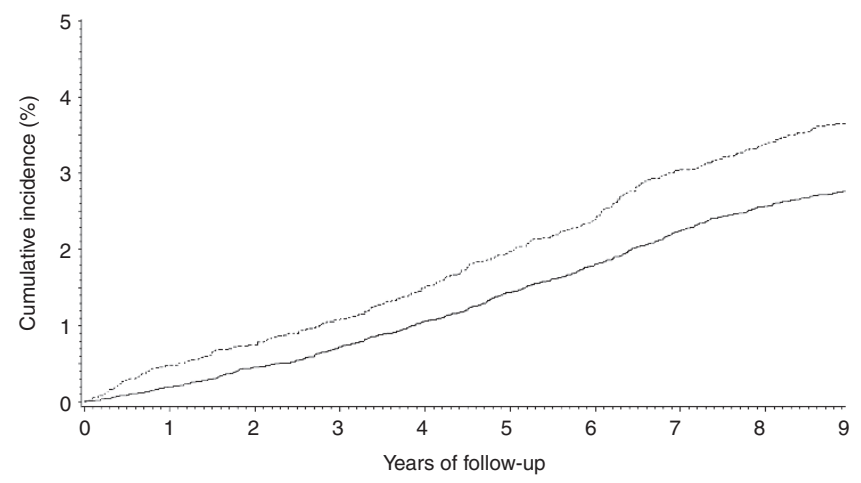

Figure 1 Cumulative incidence of neurocognitive disorders in children with and without traumatic brain injury (TBI). Cumulative incidence of attention-deficit-hyperactivity disorder (ADHD) in 10,416 children with TBI (- - ) and 41,664 without TBI (--).
$\mathrm{AHR}=1.74,95 \% \mathrm{CI}=1.11,2.72$, respectively) after adjustment for age, parental occupation, and urbanization level. However, sensitivity analysis showed no significant difference in the HRs for ADHD between the fracture and nonfracture groups stratified by age, sex, after adjustment for parental occupation, and urbanization level (Supplementary Table S3 online).

\section{DISCUSSION}

To the best of our knowledge, this is the first study comprehensively describing an association between TBI and ADHD on the basis of large-scale investigation and by considering clinical characteristics of patients in Asia. After adjusting for children's parental occupation and their residential communities' urbanization level, we observed that children with TBI had a higher ADHD risk than did those without TBI during the 9-y follow-up period. In addition, we observed that children with mild TBI and brain contusion on arrival at the hospital were more likely to develop ADHD than those who did not have a brain injury.

Findings from earlier studies are in accordance with those of our study. Allen et al. reported that TBI results in unique patterns of neurocognitive impairment, such as deficient in memory and attention abilities (23). Keenan et al. observed a significant relative risk of 1.9 compared with healthy controls, but they did not distinguish between mild and severe TBI (24).

Table 3. Adjusted HRs of ADHD for children with TBI vs. children without TBI by age, sex, and types of brain injury during the 9-y follow-up period $(n=52,080)$

\begin{tabular}{|c|c|c|c|c|c|c|}
\hline \multirow[b]{2}{*}{ Variables } & \multirow[b]{2}{*}{$N$} & \multirow[b]{2}{*}{ ADHD cases } & \multicolumn{2}{|c|}{ Crude } & \multicolumn{2}{|c|}{ Adjusted $^{\mathrm{a}}$} \\
\hline & & & $\mathrm{HR}$ & $(95 \% \mathrm{Cl})$ & $\mathrm{HR}$ & $(95 \% \mathrm{Cl})$ \\
\hline \multicolumn{7}{|c|}{ Age at first medical care for TBI } \\
\hline$\leq 4 \mathrm{y}$ & 4,594 & 369 & $1.28^{+}$ & $(1.14,1.44)$ & $1.39^{\dagger}$ & $(1.23,1.58)$ \\
\hline $5-8 y$ & 3,277 & 157 & $1.21^{*}$ & $(1.01,1.45)$ & $1.23^{*}$ & $(1.03,1.48)$ \\
\hline $9-12 y$ & 2,545 & 39 & 1.14 & $(0.78,1.65)$ & 1.12 & $(0.76,1.66)$ \\
\hline \multicolumn{7}{|l|}{ Sex } \\
\hline Male & 6,340 & 458 & $1.25^{+}$ & $(1.13,1.39)$ & $1.32^{+}$ & $(1.18,1.47)$ \\
\hline Female & 4,076 & 107 & 1.23 & $(0.99,1.54)$ & $1.31^{*}$ & $(1.03,1.65)$ \\
\hline \multicolumn{7}{|l|}{ Types of brain injury } \\
\hline No brain injury & 41,664 & 1,672 & 1.00 & (reference) & 1.00 & (reference) \\
\hline Mild brain injury & 3,605 & 150 & $1.27^{* *}$ & $(1.07,1.50)$ & $1.30^{* *}$ & $(1.10,1.53)$ \\
\hline Severe brain injury & 5,811 & 351 & $1.32^{+}$ & $(1.18,1.48)$ & $1.37^{+}$ & $(1.22,1.55)$ \\
\hline Skull fracture & 1,000 & 64 & 0.95 & $(0.74,1.23)$ & 1.10 & $(0.86,1.42)$ \\
\hline \multicolumn{7}{|c|}{ Types of severe brain injuryc } \\
\hline No brain injury & 41,664 & 1,672 & 1.00 & (reference) & 1.00 & (reference) \\
\hline Brain contusion & 988 & 53 & $1.57^{* *}$ & $(1.19,2.07)$ & $1.61^{* *}$ & $(1.22,2.13)$ \\
\hline SAH & 313 & 17 & 1.20 & $(0.74,1.93)$ & 1.30 & $(0.80,2.11)$ \\
\hline $\mathrm{EDH}$ & 240 & 20 & 1.70 & $(0.76,3.80)$ & 1.83 & $(0.82,4.11)$ \\
\hline SDH & 119 & 6 & 1.57 & $(1.00,2.44)$ & $1.74^{*}$ & $(1.11,2.72)$ \\
\hline $\mathrm{ICH}$ & 133 & 11 & 1.68 & $(0.93,3.06)$ & 1.75 & $(0.96,3.19)$ \\
\hline
\end{tabular}

ADHD, attention-deficit-hyperactivity disorder, Cl, confidence interval; TBI, traumatic brain injury.

adjustments are made for age, parental occupation and urbanization level. ${ }^{\circ}$ The interaction effect between TBI and sex was not statistically significant $(P=0.88)$. ${ }^{c}$ Brain contusion, ICD-9-CM 851; SAH, subarachnoid haemorrhage, ICD-9-CM 852.0 and 852.1; SDH, subdural haemorrhage, ICD-9-CM 852.2 and 852.3; EDH, epidural haemorrhage, ICD-9-CM 852.4 and 852.5; ICH, intracerebral haemorrhage, ICD-9-CM 853. ${ }^{*} P<0.05 ; * * 0<0.01^{\dagger} P<0.001$. 
However, two recent longitudinal investigations conducted in New Zealand and Germany, respectively, concluded that TBI is not likely to cause neurocognitive impairment in children and adolescent $(18,19)$. The discrepancy between our study and these two investigations may be explained by differences in study designs and the environmental characteristics of the target populations. The relatively small sample populations in these two studies were obtained from only a few hospitals and study centers. In contrast, our study focused on evaluating the ADHD risk in children with TBI in a large population in Taiwan, thus preventing the selection bias inherent in voluntary registration and hospital referral systems.

The characteristics of ADHD include impairment in attention, hyperactivity, inappropriate motor activity, and disruptive behavior that is inappropriate for the person's age. Our observation that ADHD frequency was higher in the children with TBI in the age group of $0-8 y$ than that in the age group of 9-12 y suggests a preinjury condition of ADHD, which might have not yet been formally diagnosed. In contrast, children aged 9-12 y were more likely to have been diagnosed with ADHD if they developed the condition. Although a delayed diagnosis of ADHD among children with TBI may confound the relationship between TBI and ADHD, our sensitivity analysis showed no significant ADHD risk in children with fractures. In addition, the cumulative incidence rate of ADHD among the children with TBI was consistently higher than that in the children without TBI during the follow-up period. Our findings strengthen the evidence that TBI is an independent risk factor for ADHD.

Our findings have valuable clinical implications for managing children with mild TBI and brain contusion. Intensive medical monitoring, support, and intervention are mostly required in the first year following a TBI event because the risk of developing neurocognitive disorders increases most rapidly in this period. Early detection of neurocognitive disorders helps children attain their full potential and prevents poor academic performance (25). Awareness of factors that lead to neurocognitive disorders and early signs or symptoms of neurocognitive disorders should be created in families, which have children with TBI through health education and interventions. Inquiry into the history of TBI in children with potential ADHD symptoms seeking medical assistance and consultation can help psychiatrists and clinical psychologists identify the high-risk group. The long-term surveillance of children with mild TBI and brain contusion for potential symptoms of ADHD would be helpful for early detection. These findings could provide clinicians to identify potential risk factors relating to $\mathrm{ADHD}$.

Using a large database has both limitations and strengths. The strengths of our study include the use of a data set that is based on a nationwide population and enabled us to follow up children with and without TBI over a long time period. The comprehensive coverage of the National Health Insurance (NHI) system and the large sample size minimized the selection and nonresponse biases and enabled us to make reasonable estimates of the effects of TBI on neurodevelopmental outcomes.
Diagnoses are validated by the National Health Insurance Administration (NHIA) in Taiwan. The NHIA requires that every hospital provide a fixed percentage of claims record from random samples to validate and preserve the quality of diagnoses. In addition, the high validity of TBI and ADHD diagnoses was confirmed by board-certified psychiatrists or physicians; however, some heterogeneity may have occurred in the assessment because of the absence of standardized instruments for diagnosis.

The study is subject to some limitations. First, a limitation imposed by the data set is the lack of details about parental and family factors (family functioning, maternal psychological distress, and social support) as well as biological factors (premature birth, birth weight, and genetic mutations). Therefore, we did not consider these factors in our research. The unavailability of these unmeasured confounders might have biased our results. Additionally, parents might pay more attention to their child who had TBI history than those without TBI exposure and bring their child to a physician when they suspect ADHD symptoms. Also, neurologists may have higher possibility to make a diagnosis when the child has TBI history. Although the diagnosis of ADHD follows the principle of clinical guidelines in Taiwan, the differential surveillance may inflate the ADHD risk and thus would be subject to some information bias. Second, although several TBI studies have reported that the locations of lesions were correlated with neurocognitive impairment $(26,27)$, we were unable to obtain detailed information of neuroimages (lesion characteristics) from Taiwan's National Health Insurance Research Database (NHIRD). Information on some external causes of TBI, such as falls, motor vehicle crashes, and violence, was also unavailable. Third, TBI outpatients who may have very minor TBI or minor symptoms were not considered in this study. We included only children with TBI who were treated at emergency medical care or inpatient care centers, potentially leading to underestimation of the number of children with TBI.

\section{Conclusions}

In summary, our results suggest that early TBI in childhood is a potential independent risk factor for ADHD. To facilitate reducing the risk of ADHD among children with TBI, a coordinated and systematic approach including a comprehensive assessment of behavioral outcomes, evolution over time postinjury, cognitive correlates, and therapeutic intervention or rehabilitation should be adopted. Additional studies are required in the future for exploring the mechanisms underlying the relationship between TBI and ADHD and developing specific diagnostic markers for identifying ADHD in children with TBI.

\section{METHODS}

\section{Database}

Data in this population-based retrospective cohort study were obtained from the NHIRD, which contains the enrollment and claims data of all beneficiaries of Taiwan's NHI program, including data on ambulatory care, records on inpatient expenditures by admissions, and a registry for beneficiaries. The single-payer NHI program was 


\section{Articles | Yangetal.}

established in 1995 and provides mandatory universal health insurance to all Taiwanese residents, covering more than $98 \%$ of the population of $\sim 23$ million people. As one of the largest databases of medical information in the world, the NHIRD provides a unique opportunity for evaluating the risk of neurocognitive disorders following TBI. This study was approved by the Taipei Medical University Institutional Review Board (TMU-JIRB-201408018). To protect patient privacy, the National Health Research Institutes manages claims data and denotes them with random identification numbers in the NHIRD. The requirement for informed consent was waived because we analyzed encrypted anonymous secondary data provided in the NHIRD.

\section{Study Population}

This study was designed as a population-based retrospective cohort study. From the NHIRD database, we selected children aged $\leq 12$ y who had visited ambulatory care centers (including the outpatient departments of hospitals or clinics) or had been hospitalized with the principal diagnosis of TBI (ICD-9-CM codes $800-804$ or $850-$ 854) between 1 January 2001 and 31 December $2002(n=11,453)$ (Figure 2). We assigned these children's first ambulatory care visit or hospitalization for TBI treatments as their medical service index use. We excluded children diagnosed with ADHD (ICD-9-CM code 314) or TBI (ICD-9-CM codes 800-804, 850-854) prior to their medical service index use $(n=586)$. Because Taiwan's NHI program was initiated in 1995, our study could not account for children diagnosed with neuronal disorders prior to 1996. In addition, we excluded children with a past medical history of preterm birth (28) (ICD-9-CM codes 765.0, 765.1) $(n=62)$, congenital anomaly (29) (ICD-9-CM codes $758,759.9)(n=27)$, mental illness (30) (ICD-9-CM codes 290-319, except 314) ( $n=183)$, epilepsy (31) (ICD-9-CM code 345) $(n=131)$, and cerebral palsy (32) (ICD-9-CM code 343$)(n=48)$ for minimizing potential confounding influence from other known risk factors for ADHD. Finally, we included 10,416 children with TBI. We further categorized TBI children into mild brain injury (ICD-9-CM code 850), severe brain injury (ICD-9-CM codes 851-854), and skull fracture (ICD-9-CM codes 800-804). Mild TBI was defined as brain concussion without structural brain damage. Severe TBI was defined as brain injury including brain contusion, SDH, epidural haemorrhage subarachnoid haemorrhage and intracranial haemorrhage. According to earlier studies $(33,34)$, we categorized the diagnoses of skull fracture combined with intracerebral hemorrhage (children with both skull fracture and intracerebral hemorrhage diagnostic codes (800-804 and 851-854) conditions, or diagnostic codes include skull fracture with intracranial injury (800.1-800.4, 800.6-800.9, etc.)) into the severe brain injury group. We furthermore defined skull fracture group as a fracture of the vault of skull, base of skull, or facial bone as well as multiple skull bone fractures without any intracerebral hemorrhage.

The non-TBI cohort was selected from the Longitudinal Health Insurance Database 2000 (LHID2000). As a subset of the NHIRD, the LHID2000 contains all original claims data for 1 million beneficiaries randomly sampled from the Registry for Beneficiaries of the NHIRD. In addition, we excluded children aged $>12$ y. Finally, we randomly selected 41,664 children without TBI (four times the number of patients with TBI) who were frequency matched with the children with TBI by sex, age $(\leq 4,5-8$, and $9-12$ y), and the year of the medical service use index. We assigned their first ambulatory service use that occurred in the year of the index healthcare use, as the medical service index use. We verified that these children had no history of neuronal disorders, TBI and ADHD prior to their medical index use since 1995. In total, we included 52,080 children (children with and without TBI). Each child was individually followed up for $9 \mathrm{y}$

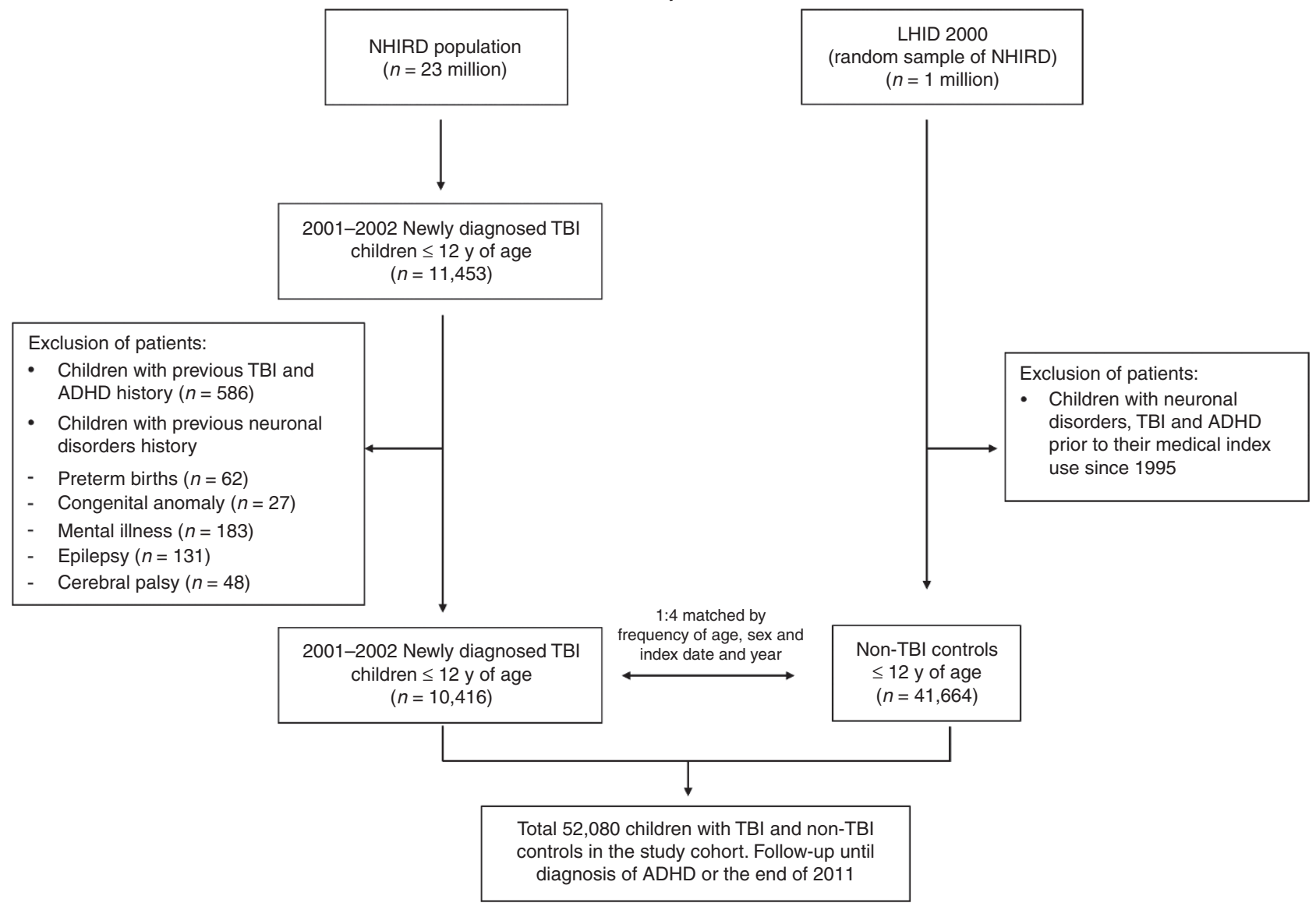

Figure 2 Flowchart of the procedure. Flowchart of case selection for children with and without traumatic brain injury (TBI) from the National Health Insurance Research Database (NHIRD) and Longitudinal Health Insurance Database 2000 (LHID2000). 
from the medical service index use to identify patients who subsequently developed ADHD (ICD-9-CM code 314). ADHD diagnosis by pediatric neurologists was based on ICD-9-CM diagnostic criteria. In Taiwan, pediatric neurologists usually provide the first diagnosis of $\mathrm{ADHD}$ according to an examination performed on observing ADHD symptoms. However, a second ADHD diagnosis is based on previous test results and clinical examination outcomes and thus provides confirmation. Recent studies reported on participants who were diagnosed at least twice for ADHD by psychiatrists and clinical psychologists to ensure diagnostic validity (35-37). Therefore, we included only patients with TBI who received two or more ADHD diagnoses to increase the diagnostic validity.

\section{Sensitivity Analysis}

To consider that ADHD may predispose children to TBI, we performed sensitivity analysis in children who received index treatment for fractures but not TBI. Children aged $\leq 12$ y diagnosed with fracture (ICD9-CM codes 805-829), including inpatients and outpatients, between 1 January 2001 and 31 December $2002(n=2,974)$ were selected from the LHID2000. We assigned their first ambulatory care visit or hospitalization for fracture treatment as their medical service index use. We excluded children with a history of preterm birth, congenital anomaly, mental illness, and cerebral palsy and those with ADHD or fracture diagnoses prior to their medical service index use $(n=326)$. In addition, children diagnosed with TBI prior to their medical service index use and 9-y follow-up duration $(n=469)$ were excluded. In the nonfracture group, children who were not diagnosed with a fracture or TBI were included. Children were randomly selected by frequency matching the fracture cohort patients with the nonfracture controls according to their age $(\leq 4,5-8$, and 9-12 y), sex, and the year of the medical service index use at a ratio of 1:4. Finally, 2,179 children with fractures but not TBI and 8,716 children without fractures were selected as cases and controls, respectively (Supplementary Figure S1 online).

\section{Statistical Analysis}

In this study, used the chi-square test for comparing differences between the cases and controls in socio-demographic characteristics such as parental occupation (white collar, employee or manual worker, self-employed (farmer, craftsman, or fisherman), and others) and the patients' urbanization level (ranging from "most urbanized" (level 1) to "least urbanized" (level 5)) at the baseline. The cumulative incidence rate was calculated using the life table method. The log-rank test was performed for evaluating the difference in the cumulative incidence between cases and controls. In addition, stratified Cox proportional hazard regression, stratified by sex, age, and follow-up year, was conducted for evaluating the association between TBI and subsequent neurocognitive outcomes during the 9 -y follow-up period. We presented HRs and their 95\% CIs as our results. Two-tailed $P$ values were calculated, and statistical significance was set at $P<0.05$. All analyses were performed using the Statistics Analysis System (SAS) statistical software, Version 9.3 (SAS Institute, Cary, NC).

\section{SUPPLEMENTARY MATERIAL}

Supplementary material is linked to the online version of the paper at http://www.nature.com/pr

\section{ACKNOWLEDGMENTS}

The dataset was obtained from the National Health Insurance Research Database provided by the Bureau of National Health Insurance, Department of Health, Taiwan, and managed by the National Health Research Institutes. The interpretations and conclusions contained herein do not represent those of the Bureau of National Health Insurance, Department of Health, or the National Health Research Institutes.

\section{STATEMENT OF FINANCIAL SUPPORT}

This study was supported in part by a grant from the Ministry of Science and Technology (MOST-104-2320-B-038-057-MY3 and MOST-1042923-B-038-001- MY3 to JY Wang), Taiwan.

Disclosure: The authors have no conflicts of interest or relevant financial relationships related to this manuscript to disclose.

\section{REFERENCES}

1. Schwartz L, Taylor HG, Drotar D, Yeates KO, Wade SL, Stancin T. Longterm behavior problems following pediatric traumatic brain injury: prevalence, predictors, and correlates. J Pediatr Psychol 2003;28:251-63.

2. Yeates KO, Armstrong K, Janusz J, et al. Long-term attention problems in children with traumatic brain injury. J Am Acad Child Adolesc Psychiatry 2005;44:574-84.

3. Roman MJ, Delis DC, Willerman L, et al. Impact of pediatric traumatic brain injury on components of verbal memory. J Clin Exp Neuropsychol 1998;20:245-58.

4. Barkley RA. Attention-deficit/hyperactivity disorder, self-regulation, and time: toward a more comprehensive theory. J Dev Behav Pediatr 1997;18:271-9.

5. Fischer M, Barkley RA, Smallish L, Fletcher K. Hyperactive children as young adults: driving abilities, safe driving behavior, and adverse driving outcomes. Accid Anal Prev 2007;39:94-105.

6. Ramos Olazagasti MA, Klein RG, Mannuzza S, et al. Does childhood attention-deficit/hyperactivity disorder predict risk-taking and medical illnesses in adulthood? J Am Acad Child Adolesc Psychiatry 2013;52: 153-162.e4.

7. Konrad K, Gauggel S, Manz A, Schöll M. Inhibitory control in children with traumatic brain injury (TBI) and children with attention deficit/ hyperactivity disorder (ADHD). Brain Inj 2000;14:859-75.

8. Max JE, Lansing AE, Koele SL, et al. Attention deficit hyperactivity disorder in children and adolescents following traumatic brain injury. Dev Neuropsychol 2004;25:159-77.

9. Schachar R, Levin HS, Max JE, Purvis K, Chen S. Attention deficit hyperactivity disorder symptoms and response inhibition after closed head injury in children: do preinjury behavior and injury severity predict outcome? Dev Neuropsychol 2004;25:179-98.

10. Adeyemo BO, Biederman J, Zafonte R, et al. Mild traumatic brain injury and ADHD: a systematic review of the literature and meta-analysis. J Atten Disord 2014;18:576-84.

11. Andrews PJ, Piper IR, Dearden NM, Miller JD. Secondary insults during intrahospital transport of head-injured patients. Lancet 1990;335: 327-30.

12. Babikian T, Asarnow R. Neurocognitive outcomes and recovery after pediatric TBI: meta-analytic review of the literature. Neuropsychol 2009;23:283-96.

13. Anderson V, Godfrey C, Rosenfeld JV, Catroppa C. Predictors of cognitive function and recovery 10 years after traumatic brain injury in young children. Pediatr 2012;129:e254-61.

14. Max JE, Arndt S, Castillo CS, et al. Attention-deficit hyperactivity symptomatology after traumatic brain injury: a prospective study. J Am Acad Child Adolesc Psychiatry 1998;37:841-7.

15. Max JE, Schachar RJ, Levin HS, et al. Predictors of attention-deficit/hyperactivity disorder within 6 months after pediatric traumatic brain injury. J Am Acad Child Adolesc Psychiatry 2005;44:1032-40.

16. Slomine BS, Salorio CF, Grados MA, Vasa RA, Christensen JR, Gerring JP. Differences in attention, executive functioning, and memory in children with and without ADHD after severe traumatic brain injury. J Int Neuropsychol Soc 2005;11:645-53.

17. Wassenberg R, Max JE, Lindgren SD, Schatz A. Sustained attention in children and adolescents after traumatic brain injury: relation to severity of injury, adaptive functioning, ADHD and social background. Brain Inj 2004;18:751-64.

18. McKinlay A, Grace R, Horwood J, Fergusson D, MacFarlane M. Adolescent psychiatric symptoms following preschool childhood mild traumatic brain injury: evidence from a birth cohort. J Head Trauma Rehabil 2009;24:221-7.

19. Petersen C, Scherwath A, Fink J, Koch U. Health-related quality of life and psychosocial consequences after mild traumatic brain injury in children and adolescents. Brain Inj 2008;22:215-21.

20. Massagli TL, Fann JR, Burington BE, Jaffe KM, Katon WJ, Thompson RS. Psychiatric illness after mild traumatic brain injury in children. Arch Phys Med Rehabil 2004;85:1428-34. 


\section{Articles | Yangetal.}

21. Chou IC, Lin CC, Sung FC, Kao CH. Attention-deficit-hyperactivity disorder increases risk of bone fracture: a population-based cohort study. Dev Med Child Neurol 2014;56:1111-6.

22. Thomas CR, Ayoub M, Rosenberg L, Robert RS, Meyer WJ. Attention deficit hyperactivity disorder \& pediatric burn injury: a preliminary retrospective study. Burns 2004;30:221-3.

23. Allen DN, Leany BD, Thaler NS, Cross C, Sutton GP, Mayfield J. Memory and attention profiles in pediatric traumatic brain injury. Arch Clin Neuropsychol 2010;25:618-33.

24. Keenan HT, Hall GC, Marshall SW. Early head injury and attention deficit hyperactivity disorder: retrospective cohort study. BMJ 2008;337:a1984.

25. Glascoe FP. Early detection of developmental and behavioral problems. Pediatr Rev 2000;21:272-9; quiz 280.

26. Babikian T, Freier MC, Tong KA, et al. Susceptibility weighted imaging: neuropsychologic outcome and pediatric head injury. Pediatr Neurol 2005;33:184-94.

27. Ylvisaker M, Feeney T. Pediatric brain injury: social, behavioral, and communication disability. Phys Med Rehabil Clin N Am 2007;18:133-44, vii.

28. Lindström K, Lindblad F, Hjern A. Preterm birth and attention-deficit/ hyperactivity disorder in schoolchildren. Pediatr 2011;127:858-65.

29. Halmøy A, Klungsøyr K, Skjærven R, Haavik J. Pre- and perinatal risk factors in adults with attention-deficit/hyperactivity disorder. Biol Psychiatry 2012;71:474-81.
30. Robison LM, Sclar DA, Skaer TL, Galin RS. Treatment modalities among US children diagnosed with attention-deficit hyperactivity disorder: 199599. Int Clin Psychopharmacol 2004;19:17-22.

31. Dunn DW, Austin JK, Harezlak J, Ambrosius WT. ADHD and epilepsy in childhood. Dev Med Child Neurol 2003;45:50-4.

32. Shank LK, Kaufman J, Leffard S, Warschausky S. Inspection time and attention-deficit/hyperactivity disorder symptoms in children with cerebral palsy. Rehabil Psychol 2010;55:188-93.

33. Bazarian JJ, Veazie P, Mookerjee S, Lerner EB. Accuracy of mild traumatic brain injury case ascertainment using ICD-9 codes. Acad Emerg Med 2006;13:31-8.

34. Yeh CC, Chen TL, Hu CJ, Chiu WT, Liao CC. Risk of epilepsy after traumatic brain injury: a retrospective population-based cohort study. J Neurol Neurosurg Psychiatry 2013;84:441-5.

35. Chen MH, Chen YS, Hsu JW, et al. Comorbidity of ADHD and subsequent bipolar disorder among adolescents and young adults with major depression: a nationwide longitudinal study. Bipolar Disord 2015;17:315-22.

36. Chou PH, Lin CC, Lin CH, Loh el-W, Chan CH, Lan TH. Prevalence of allergic rhinitis in patients with attention-deficit/hyperactivity disorder: a population-based study. Eur Child Adolesc Psychiatry 2013;22:301-7.

37. Shyu CS, Lin HK, Lin CH, Fu LS. Prevalence of attention-deficit/hyperactivity disorder in patients with pediatric allergic disorders: a nationwide, population-based study. J Microbiol Immunol Infect 2012;45:237-42. 